\title{
DE SENDEROS A PAISAJES: PAISAJES DE LAS MOVILIDADES DE UNA COMUNIDAD MAPUCHE EN LOS ANDES DEL SUR DE CHILE
}

\author{
FROM TRAILS TO LANDSCAPES: LANDSCAPES OF THE MOBILITIES OF A \\ MAPUCHE COMMUNITY IN THE SOUTHERN ANDES OF CHILE
}

\author{
Viviana Huiliñir-Curío ${ }^{1}$
}

\begin{abstract}
Este artículo examina los paisajes de las movilidades de una comunidad mapuche situada en la comuna cordillerana de Curarrehue, zona reconocida por las múltiples redes de intercambio y tráfico comercial transfronterizo mapuche, en los Andes del sur de Chile. La metodología de investigación que orienta este trabajo es cualitativa, con enfoque etnográfico y narrativo. A partir de los resultados de la investigación, se distinguieron tres paisajes asociados a rutas y movilidades específicas de la comunidad: el paisaje de Mongolluko, el paisaje de Añihuerraqui y el paisaje de Trankura. Finalmente, se concluye que los paisajes de las movilidades mapuches se reproducen a través de las narrativas de las movilidades pasadas y presentes respecto a sitios y rutas específicas, cuyas prácticas y discursos desafían y subvierten los efectos de las intervenciones de la agencia estatal en la cordillera de los Andes.
\end{abstract}

Palabras claves: paisajes, movilidades mapuches, Lof Trankura, cordillera de los Andes, Curarrehue.

This article examines the landscapes of the mobilities of a Mapuche community located in the Andean commune of Curarrehue, recognized by the multiple networks of Mapuche exchange and cross-border commercial traffic in the southern Andes of Chile. The research methodology is qualitative with an ethnographic and narrative approach. Based on the results of the research, three landscapes associated with specific routes and mobilities of the community were distinguished: the Mongolluko landscape, the Añihuerraqui landscape and the Trankura landscape. It is concluded that the landscapes of the Mapuche mobilities are reproduced through narratives of past and present mobilities with regard to specific sites and routes, whose practices and discourses challenge and subvert the effects of State agency on the Andes mountains.

Key words: Landscapes, Mapuche mobilities, Lof Trankura, Andes mountains, Curarrehue.

Hasta principios del siglo XX, la movilidad mapuche en los Andes del sur de Chile brindó continuidad territorial a un vasto territorio indígena comprendido entre la Araucanía y las Pampas argentinas. Los mapuches se desplazaban con mucha frecuencia entre ambos lados de la cordillera de los Andes por rutas cordilleranas específicas, motivados por razones comerciales, lazos de parentesco y alianzas políticas y militares (Bello 2000, 2011; Bengoa 2000; Capellà 2009; Curilaf 2015; Godoy 2014; Gundermann et al. 2009a, 2009b; Pinto 1996; Zavala 2011). No obstante, a finales del siglo XIX, diversas estrategias de ocupación del Estado chileno en territorio mapuche trajeron consigo el trazado de límites nacionales que transformaron la cordillera de los Andes en frontera política (Benedetti y Salizzi 2014; Núñez et al. 2013) a través del control sobre el uso y acceso de los pasos cordilleranos y la creación de parques y reservas nacionales, fragmentando el territorio indígena y cambiando las pautas de movilidad mapuche entre la Araucanía y Las Pampas.

La movilidad mapuche por determinados senderos o rüpü -en mapudungun- fueron importantes no tan solo en términos pragmáticos; también han constituido un sistema de prácticas, conexiones y significados otorgados a un paisaje ritualizado y marcado por el tránsito humano (Bello 2014; Godoy 2014; Huiliñir-Curío 2015) que continúa vigente en la memoria social, resistiendo y desafiando las múltiples intervenciones estatales (Huiliñir-Curío 2016). Por lo tanto, en un contexto de avance y consolidación del Estado chileno en la cordillera de los Andes, cobran relevancia las narrativas sobre la movilidad mapuche en los márgenes nacionales en tanto productora

\footnotetext{
${ }^{1}$ Centro Internacional de Estudios de La Patagonia (CIE-Patagonia), Núcleo de Ciencias Sociales y Humanidades, Universidad de La Frontera, Temuco, Chile. viviana.huilinir@ufrontera.cl
} 
de paisajes que conectan memorias, significados y experiencias en tensión con los procesos estatales de colonización, privatización y sus renovadas formas de despojo (Di Giminiani 2012; Huiliñir-Curío 2016).

Aproximaciones recientes al estudio de la movilidad como fenómeno antropológico y geográfico, la conciben como un acto corporal que nos permite habitar el mundo y relacionarnos con el territorio (Careri 2002), creando paisajes e historias en distintas escalas de desplazamientos situados en determinados contextos históricos (Cresswell 2006). Dado que la movilidad configura y es configurada por procesos sociales, económicos y políticos, es posible asumirla como una categoría analítica de diversos mecanismos de control sobre la sociedad presente y pasada, incluyendo discursos, prácticas, infraestructuras y tecnologías capaces de impulsar o frenar el movimiento (Urry 2007). A su vez, la movilidad, como práctica significativa y significante (Tomasi 2013) ofrece un terreno rico de narrativas sujetas a una historicidad que devela sus cambios y permanencias enmarcadas y reguladas por relaciones de poder (Cresswell 2010).

Pese al progresivo interés de las ciencias sociales por esta temática, ha tenido un limitado acercamiento a las movilidades indígenas en contextos coloniales contemporáneos, cuyas narrativas y experiencias resultan relevantes para expandir nuestros marcos de entendimientos sobre procesos sociales e históricos mayores. Tomando en cuenta que el pueblo mapuche ha sido históricamente sujeto de procesos hegemónicos de dominación, los relatos y vivencias asociadas a la movilidad en la cordillera son aspectos que merecen nuestra atención cuando se analizan las trayectorias e implicancias de los actuales conflictos en un sentido más amplio. Al respecto, destacan los trabajos de Bello (2000, 2011), Curilaf (2015), Gundermann et al. (2009a, 2009b) y Pinto (1996), quienes examinan la movilidad transfronteriza del pueblo mapuche en Chile desde una mirada historiográfica. Otros aportes son los realizados por Godoy (2014), Huiliñir-Curío (2015), Huiliñir-Curio y MacAdoo (2014) y Salazar et al. (2017) quienes analizan las prácticas de movilidad mapuche contemporánea en la zona cordillerana de la región del Biobío, La Araucanía y Los Ríos.

Con el propósito de contribuir a las discusiones sobre movilidad y paisaje desde una perspectiva indígena, este artículo examina las formas en que los cambios de los desplazamientos mapuches a lo largo de rutas y pasos cordilleranos, influyen en la producción de paisajes de una comunidad mapuche de la comuna Curarrehue en la región de La Araucanía, Chile (Huiliñir-Curío 2016). La noción de paisaje resulta útil en la medida que nos acerca a las dimensiones más subjetivas de la movilidad mapuche basada en la experiencia encarnada que dota de sentido a sitios específicos (Careri 2002; Tilley 1994) cuyos significados e historias son compartidas colectivamente (Di Giminiani 2012). Esta perspectiva centrada en la experiencia en el paisaje como proceso encarnado, le brinda una importancia ontológica al concepto, relacionando lugares, rutas, movimientos, cuerpos y narrativas desde nuestros desplazamientos en el espacio como un todo (Ingold 2000). Por otro lado, la dimensión discursiva del paisaje relacionada con su materialidad, abre la posibilidad de atender los rasgos emocionales presentes en dicha relación (Skewes et al. 2011) mientras que, por medio de la percepción, es posible registrar los cambios temporales del entorno (Giminiani 2012).

La primera parte del artículo describe el área de estudio y las estrategias metodológicas utilizadas para el desarrollo de este estudio. La segunda analiza los paisajes asociados a rutas y las narrativas de las movilidades del Lof Trankura, ordenados en función de una tipología de movilidades pasadas y presentes que dan cuenta de un uso más acotado del espacio en la actualidad, producto de las intervenciones del Estado nación chileno en este territorio: el paisaje de Mongolluko, el paisaje de Añihuerraqui y el paisaje de Trankura. En la tercera sección se discuten de manera detallada las dimensiones materiales y discursivas que definen los paisajes producidos por las movilidades del Lof Trankura. Finalmente se concluye que los paisajes de las movilidades mapuches son producidos por las narrativas de las movilidades pasadas y presentes que desafían y resisten la intervención estatal sobre las formas de habitar el territorio en la cordillera.

\section{Paisajes de las Movilidades Mapuches del Lof Trankura}

Para muchos pueblos indígenas, como los mapuches, el paisaje encarna nociones de una naturaleza que forma parte de un proceso vivo constituyente del mundo habitado (Di Giminiani y Fonck 2015; Ingold 2000). Este carácter relacional del paisaje da cuenta de un proceso de interacción social entre entidades humanas y no humanas (Di Giminiani y Fonck 2015; Skewes y Guerra 2016; Skewes et al. 2014) que le otorga vitalidad y constante movimiento (Salazar et al. 2017). Para el caso del pueblo mapuche, los paisajes contienen la memoria de la comunidad y a su vez, son la memoria práctica de las comunidades, cuyas relaciones simbióticas permiten que la comunidad se reconozca en el paisaje (Skewes et al. 2011). Por lo tanto, el paisaje contextualiza el presente e influirá en las reacciones de una comunidad frente a variadas intervenciones externas (Skewes y Guerra 2016). Es por esta razón que cobra relevancia 
explorar qué sucede cuando la movilidad mapuche -productora de paisajes- es afectada por procesos derivados de la agencia estatal.

El área considerada para este estudio es el Lof Trankura, organización social tradicional mapuche emplazada en el sector sureste del valle del río Trancura, en la comuna de Curarrehue, región de la Araucanía, cercana a los pasos fronterizos de Añihuerraqui, Paimún y Mamuil Malal (Figura 1). Geográficamente, el valle del Trankura está conformado por grandes cordones montañosos caracterizados por la existencia de sitios de veranada o valles altos, bosques de araucarias o pewen (Araucaria araucana), cascadas y volcanes. Dada su condición de boquete, fue ampliamente utilizado por los mapuches que viajaban hacia las pampas argentinas desde principios del siglo XIX hasta mediados del siglo XX motivados por la caza, recolección de frutos, comercio e intercambio de productos como ganado y yerba mate (Curilaf 2015; Godoy 2014).

Un hito significativo de la historia del Lof Trankura ocurre a finales del siglo XIX, durante la ocupación militar de los Estados nacionales chileno y argentino en territorio mapuche, que provocó la migración forzada de numerosas familias mapuches desde Panguipulli, localidad situada al sur de La Araucanía, hasta el valle del Trancura (Barrientos 2015). Tras la huida a lo largo de la cordillera de los Andes, fueron radicados en el valle del Trancura el Longko Camilo Coñoequir Lloftunekul y su grupo familiar a través de un Título de Merced entregado el año 1909 y que concede reconocimiento legal de la propiedad mapuche por parte del Estado nación chileno (Barrientos 2015). Una vez otorgado el Título de Merced, Lloftunekul asignó tierras a los integrantes de su grupo familiar y organizó el territorio de acuerdo a una toponimia específica en función de las lógicas mapuches e iniciando un proceso de reapropiación de este nuevo espacio (Barrientos 2015).

Actualmente el Lof Trankura sobresale por múltiples iniciativas de turismo comunitario gestionadas y lideradas por sus integrantes que lo han posicionado como un referente regional. No obstante, desde el 2009 aproximadamente, el Lof Trankura se ha enfrentado a la construcción de una mini central hidroeléctrica de pasada, cuyo titular es la empresa GDT Negocios S.A. La construcción de esta central de pasada fue aprobada el año 2015 por la Comisión de Evaluación de Medioambiente del Estado chileno, la cual se instalará en el curso superior del estero Añihuerraqui o "Pichitrankura", cuya área de influencia incluye el camino de una antigua ruta que conecta al Lof Trankura con el paso fronterizo Añihuerraqui y un espacio de veranada que ahora pertenece a particulares.
La aprobación de la mini central hidroeléctrica ha sido controversial para las familias de Lof Trankura, quienes denuncian la vulneración de lugares sagrados y del estero Añihuerraqui, del cual han dependido desde que habitan el valle (Castro et al. 2016). Estas tensiones se han profundizado con el cercamiento del acceso al camino hacia el paso Añihuerraqui. Estos procesos de privatización impulsados por el Estado nacional chileno, no solo implica restricciones en la ocupación de espacios específicos y sus recursos, sino que también penetran en las dimensiones más subjetivas de las familias mapuches del Lof Trankura, quienes apelan a la memoria social para reivindicar la defensa del territorio y sus lugares sagrados.

Considerando que las técnicas cualitativas se orientan a captar, interpretar y analizar los aspectos significativos de los individuos o grupos a través de la libre manifestación de sus intereses, creencias y deseos (Hernández et al. 2006), esta investigación consideró métodos etnográficos y biográficos para la recolección de datos primarios durante un trabajo de campo realizado entre enero y julio de 2016, que incluyó entrevistas semi-estructuradas, relatos de vida, notas de campo y recorridos guiados por rutas y pasos, todo esto bajo un acuerdo previo con las familias del Lof Trankura y la solicitud de un consentimiento informado a cada participante, cuyas identidades han sido resguardadas debido a los conflictos suscitados por la central Añihuerraqui en el territorio. En total, se realizaron nueve entrevistas individuales, un relato de vida y dos entrevistas grupales a diferentes miembros del Lof Trankura, que fueron transcritas, codificadas y analizadas en torno a tres grandes temas: movilidad, narrativas del paisaje y fronterización de la cordillera de los Andes. Los datos permitieron construir una tipología de movilidades específicas asociadas a momentos y lugares concretos que revelan un proceso de acotamiento de los desplazamientos en el territorio (Figura 2). A su vez, se identificaron paisajes asociados a rutas específicas en función de las narrativas de las movilidades a lo largo de la cordillera de los Andes y que responden a escalas y contextos políticos, económicos y sociales particulares.

De esta manera, desde de los testimonios relacionados con la movilidad mapuche en la cordillera y con apoyo de fuentes de tipo secundaria, fue posible brindar un marco interpretativo para explorar la producción paisajes en torno a rutas que conectaban la vertiente oriental y occidental de la cordillera de los Andes: la ruta por el paso Añihuerraqui, la ruta por el paso Paimún (Figura 3). Al mismo tiempo, otorgaron la posibilidad de explorar las nuevas pautas de movilidad cotidiana hacia el interior del valle del Trancura. 


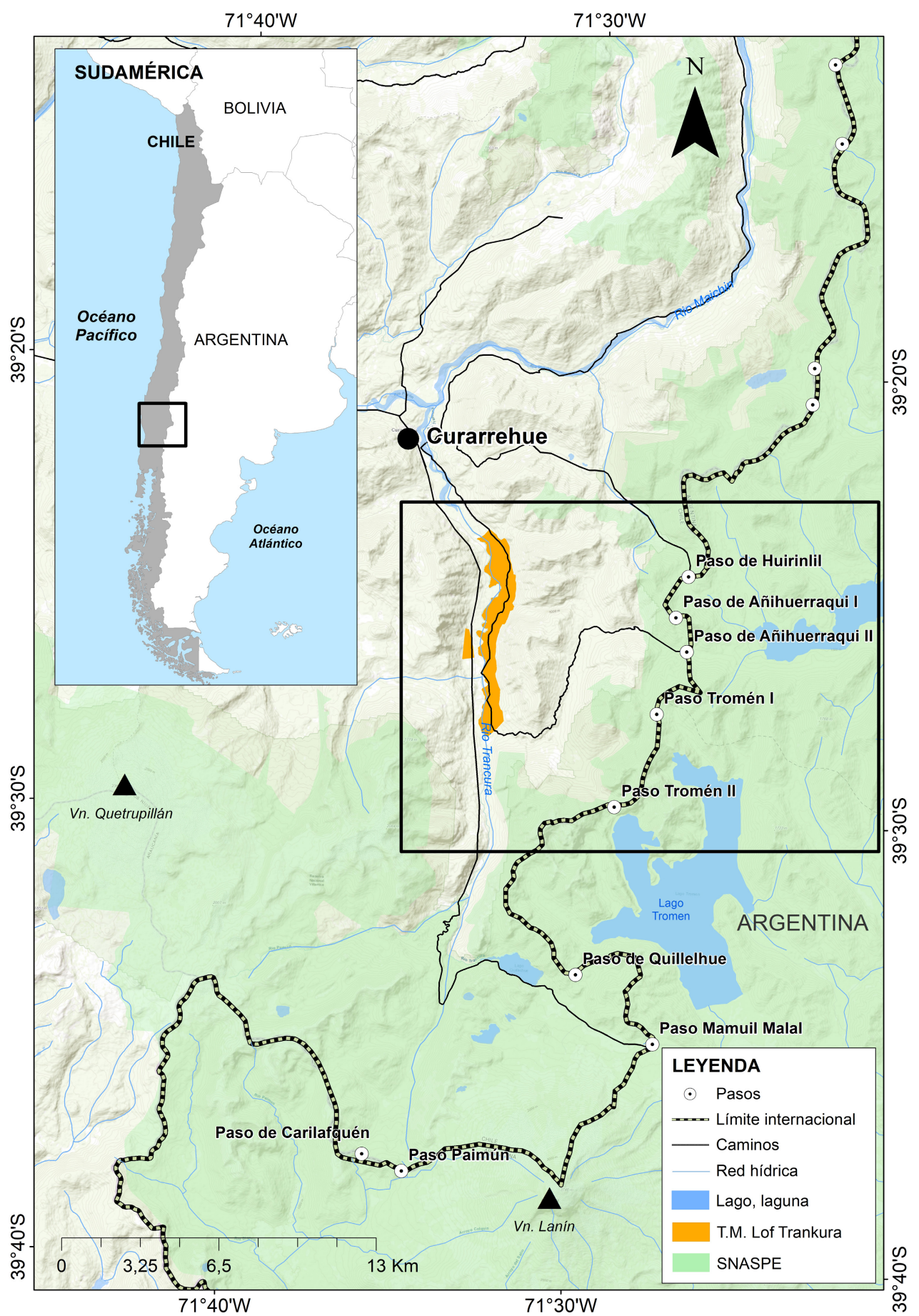

Figura 1. Lof Trankura. Localización aproximada. Elaboración propia.

Lof Trankura. Approximate location. Map credit: author. 


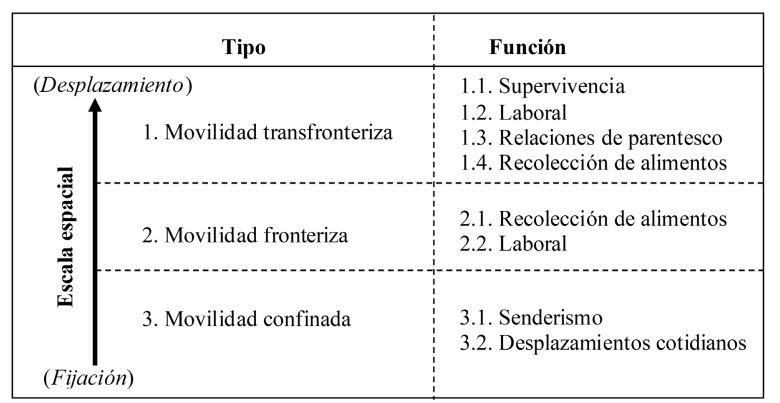

Figura 2. Tipología de movilidades del Lof Trankura. Elaboración propia. Typology of Lof Trankura mobilities. Credit: author.

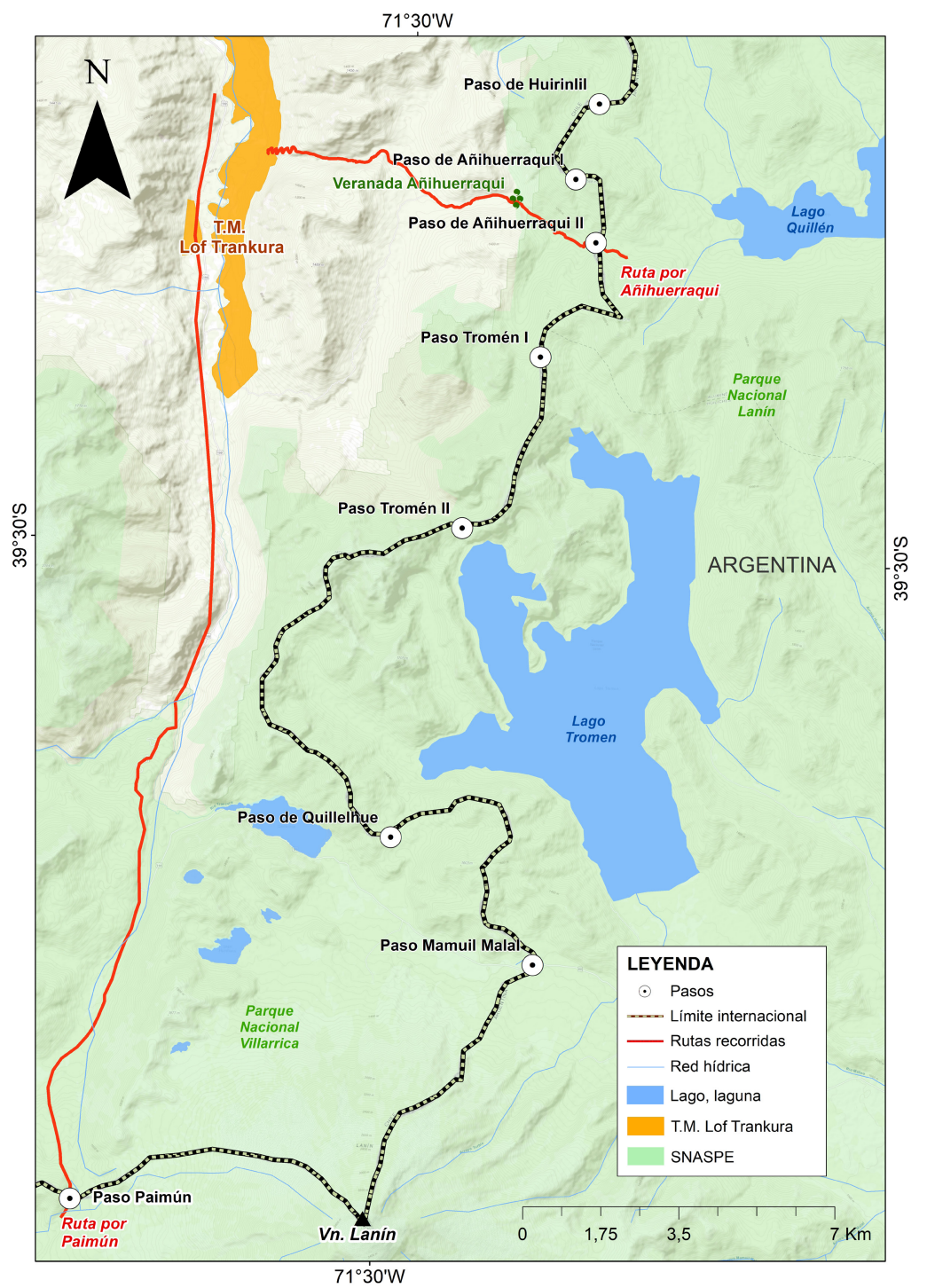

Figura 3. Rutas de Lof Trankura, valle del río Trancura. Elaboración propia. Lof Trankura routes, Trancura River Valley. Map credit: author. 


\section{Paisaje de Mongulluko}

La ruta hacia el paso Paimún, situado a 1.424 $\mathrm{msm}$, forma parte del valle río Momolluco, contenido entre los faldeos noroccidentales del volcán Lanín y un extenso cordón cordillerano por el lado occidental, del que emerge el cerro Porue. En el valle destacan los bosques de araucarias, remanentes de coigüe (Nothofagus dombeyi), quila (Chusquea quila), lenga (Nothofagus pumilio) y ñirre (Nothofagus antarctica), donde también sobresalen varios cuerpos de agua como la laguna Huenfuica, la laguna El Plato, la laguna Perdida y laguna Verde, predominando la presencia del río Momolluco por sobre todos ellos (Figura 4).

La ruta responde a un tipo de movilidad transfronteriza, motivada por razones laborales, recolección de alimentos y de parentesco. En efecto, esta ruta es conocida para muchas personas del Lof Trankura, particularmente para aquellas que superan los 50 años, quienes acudían al valle recolectando piñones o ngüilliu -fruto de la araucaria- durante largos periodos de tiempo. El traslado hacia este espacio implicaba una preparación previa a nivel familiar, que culminaba con la construcción de ranchos estacionales, hechos con quila y materiales ligeros. En el presente, este valle forma parte del Parque Nacional Villarrica, creado el año 1940, y su acceso ha sido intervenido de tal forma de evitar la entrada de vehículos, siendo exclusivo el uso para personas visitantes del parque. Además, la antigua huella que continuaba por el valle del Trancura fue interceptada por la ruta internacional $\mathrm{CH}-199$, que divide la ruta y dificulta el acceso a esta zona.

La toponimia nos acerca a los contenidos del paisaje de la ruta, resaltando muchas cualidades, funciones y atributos míticos asignados a ciertos lugares que destacan sobre otros. Un ejemplo de lo anterior es la toponimia del paso Paimún, cuyo nombre original es "Rüpaimün" que quiere decir "pasaron por ahí" o "pasando" (Curilaf 2015). Este nombre reafirma la condición de portal de este boquete que fue ampliamente usado por los mapuches que cruzaban la cordillera desde y hacia Argentina por el faldeo noroccidental del volcán Lanín, el cual brindaba mayor seguridad para el tránsito a lo largo de la cordillera. Lo mismo sucede con el volcán Lanín, nombrado por algunos participantes como "Awelñamko" que significa "donde revolotea el águila" (Curilaf 2015). El águila es un ave totémica mencionada recurrentemente en las rogativas de los mapuches del Lof Trankura cuya presencia proporciona un sentido de vitalidad al volcán:

Awelñamko... Eso como es un escorial que está formado un volcán ahí. Entonces se dan cuenta que siempre había un águila que estaba preocupado por viajar e irse a las partes montañosas y entonces gritaba: "kau-kau, kau-kau, kau-kau". Y seguía revoloteando. Por eso le pusieron Awelñamko pillán. Y nosotros cuando nguillatukamos lo nombramos así (entrevista 8: hombre, 70 años).

El cerro Porue (1.448 msm) llamado por los mapuches del Lof Trankura como 'Purruwe', es otro hito geográfico del paisaje destacado por los participantes, cuyo nombre significa "lugar donde se hace purrun". El purrun es una danza de tipo ritual religiosa, practicada durante la ceremonia del nguillatun. En el caso de Porue, quienes bailan o purrukan son el ngen mawida (cuidador de los bosques que están en los montes) y el ngen pewenentu (cuidador de los bosques de Araucaria) del Lof Trankura. Es importante señalar la rica de geografía imaginaria asociada a los paisajes, de la que emergen elementos como los ngen o seres espirituales que cuidan diversos espacios y a quienes es necesario pedir permiso antes de traspasar algún sitio que resguardan (Grebe 1993). Otros ngen que forman parte de este paisaje son: ngen rüpü (cuidador de las huellas o senderos), ngen ko (cuidador del agua) y ngen wingkul (cuidador de los cerros).

Los entrevistados hacen mención a la existencia de un ngen mawiza y ngen pewenentu conocidos como faimpu, que habitan en los bosques de araucarias que rodean el volcán Lanín, y que posee la forma humana de un anciano o una anciana:

Por ejemplo, si vamos a los pinos el ngen mawiza, el ngen pewenentu mawiza se llama faimpи y por lo general siempre el piñonero ve un faimpu: un viejito, una viejita con un saco al hombro llevándose piñones. Entonces es por eso que siempre hay que pedir que entregue abundancia de producto, de pewen... nguillio (entrevista 8: hombre, 70 años).

Este ngen es muy temido por los hombres y mujeres mapuches con más edad del Lof Trankura, quienes deben recoger todos aquellos piñones o nguillio que ofrecen las araucarias sin hacer distinciones entre los tamaños del piñón, ya que esto podría desencadenar la molestia del faimpu. Este aspecto del mundo mapuche es muy mencionado durante las entrevistas, en las cuales relatan sobre cómo se comunican con los ngen de cada lugar por medio del nguilllatukeo o rogativa, que es una manera de conectarse con ellos para pedirles protección y en otras instancias demostrar agradecimiento por el alimento resguardado y cedido por los ngen a los mapuches. 


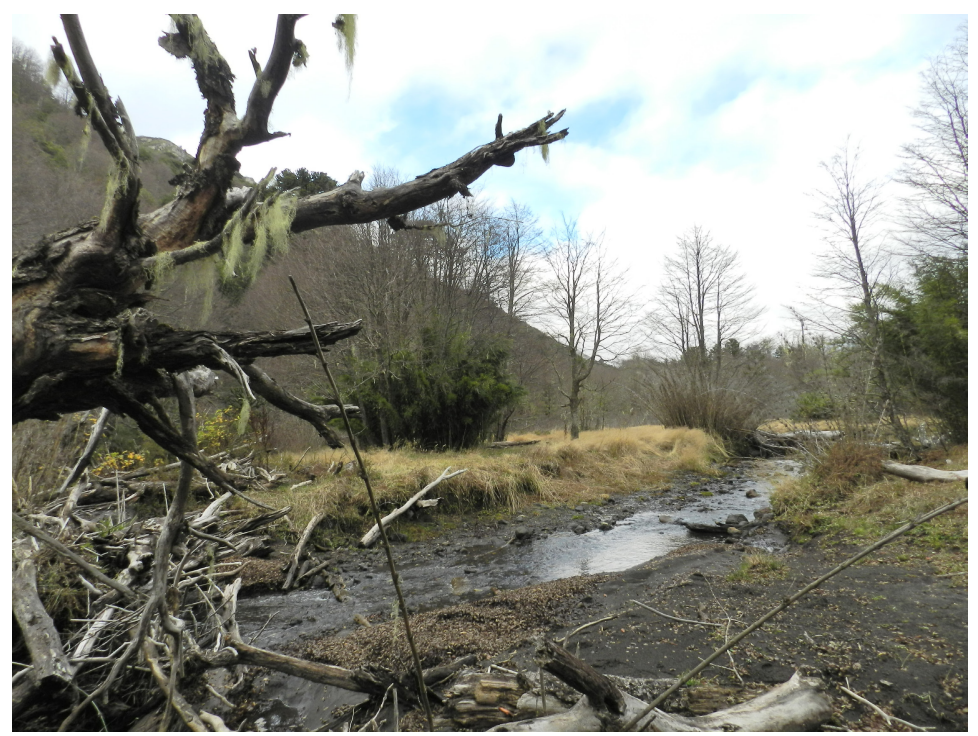

Figura 4. Valle del río Momolluco. Registro personal de la autora.

Momolluco River Valley. Photo credit: author.

Otro elemento interesante de mencionar se relaciona con los vínculos construidos a partir de las narrativas de los desplazamientos con las generaciones más jóvenes del Lof Trankura. La experiencia vivida en esta ruta se traspasa a las generaciones posteriores, quienes se sienten familiarizados con este valle y distinguen las marcas sobre la materialidad del paisaje que forman parte de las huellas del pasado dejadas por las familias mapuches que compartían en Mongolluko.

Yo nunca vi, yo no alcancé a vivir, digamos la vivencia que tuvo mi mamá, ahí, donde iban a buscar piñones, iban a acampar por una semana, un mes, dependiendo de cómo estaba el clima, el tiempo (...) Pero yo me daba el tiempo de recorrer. He visto cómo están los lugares donde estaban los rüpü de antes, que todavía quedan vestigios. Lugares donde acampaban y las rutas por donde pasaban los carros (entrevista 12: hombre, 22 años).

Ciertamente los paisajes surgidos desde la experiencia directa en esta ruta y sus sitios relevantes permiten trazar vínculos emocionales y afectivos y disponer de mecanismos de anclaje al lugar (Lindón 2006) en mapudungun, que repercuten en la percepción sobre los mismos, que se ven enfrentadas a las transformaciones materiales de los propios paisajes.

\section{Paisaje de Añihuerraqui}

La ruta hacia el paso Añihuerraqui fue otro paisaje posible de distinguir de la movilidad de tipo transfronteriza de las familias mapuches del Lof Trankura. El recorrido se realizaba a través del 'camino maderero', vía de ripio desde el cual se asciende por la ladera sur del cerro Pünowemañke, siguiendo el movimiento natural de la quebrada del estero Pichitrankura, que se encuentra entre el cerro Pünowemañke y el cerro Peñewe. El camino está en buen estado, pero tiene acceso restringido para el Lof, debido a que los dueños del predio mantienen cercado el sitio usado explosivamente por la empresa GTD Negocios S.A y sus maquinarias para la construcción de la central de paso Añihuarraqui en el nacimiento del estero.

Originalmente, el camino maderero formaba parte de una ruta usada por los mapuches del valle del Trancura para cruzar hacia Argentina por el paso de Añihuerraqui en busca de piñones o para desplazarse con ganado hacia las veranadas situadas en los valles limítrofes con Argentina. Años más tarde, el camino maderero fue habilitado para el tránsito de animales de carga, como bueyes, que transportaban leña desde firmas madereras dedicadas a la explotación de raulí (Nothofagus alpina) y coligüe (Chusquea culeou) en el sector de Añihuerraqui durante los años treinta, en terrenos que eran mayoritariamente propiedad estatal y de colonos nacionales (Curilaf 2015). Si bien las fábricas de maderas cerraron durante la década de 1970, la explotación maderera continúa en el sector de Añihuerraqui, en propiedades y ex propiedades de los colonos chilenos que colindan con el Parque Nacional Villarrica y que aún extraen madera nativa, pero en menor escala.

Este camino también forma parte de un paisaje asociado a la infancia, dado que les permitía acceder 
a una veranada que, actualmente, es propiedad de particulares. En efecto, el nombre de esta veranada llamada 'Añihuerraqui' guarda relación con las narrativas de un paisaje 'limpio' y 'tranquilo', en el cual anidan las bandurrias, aves características del sur de Chile. Pese a que no son propietarios legales de ese espacio, el camino forma de la memoria compartida respecto a los desplazamientos a través de este paso. Es por ello también el malestar por la intervención de la empresa sobre su acceso, generando tensiones entre los habitantes del sector: "ese era de uso público porque esto era de mi abuelita. Por ahí salía toda la gente. Gente que era de Villarrica, gente que era de otros lugares vienen y pasaban por ahî' (entrevista 6: mujer, 87 años).

El camino continúa por la otra ribera del Pichitrankura, una vasta pradera con renovales de coigües y roble pellín. Este valle altoandino es un espacio de veranada, ocupado según lo narrado por algunas familias mapuches del Lof Trankura en época estival. Nociones de nostalgia por la libertad de circular en estos espacios son explicadas por la inexistencia de cercas o límites físicos, que son los actuales dispositivos de control de la propiedad de la tierra impuestos durante el periodo de neoliberalización de la economía chilena a finales de la década de 1980.

En los límites visuales de la pradera, se asoman los límites físicos del Parque Nacional Villarrica. Traspasada la entrada del parque, el camino continúa nítido y es posible observar madera apilada, restos abandonados de la antigua actividad maderera dentro del parque y que culmina en el periodo de Dictadura Militar (Figura 5). La ruta y su geografía se tornan similares al de una selva deshabitada. Y el camino, sin mantención, está bloqueado por grandes troncos de madera que yacen recostados a lo ancho de éste, interrumpiendo el tránsito.

El camino va desapareciendo, pero el guía del camino comienza a rastrear las marcas puestas en varios puntos que indican la ruta. Pese a que el guía, así como muchas personas mapuches del Lof Trankura, dejaron de transitar esta huella para ir al lago Quillén, localizado en territorio argentino, aún mantienen ciertas destrezas respecto a cómo leer las referencias sobre un territorio en el cual es fácil perderse. Y estas estrategias para desplazarse en la espesura de la selva cordillerana no han sufrido muchas variaciones, dado que los códigos se siguen manteniendo. Esto coincide con Connerton (1989) quien afirma que nuestra experiencia del presente depende, en gran medida, de nuestro conocimiento del pasado.

El paisaje de esta ruta, en este tramo, es dotado de un sentido asociado a 'lo masculino' por un lado, puesto que eran principalmente hombres quienes recorrían esta ruta, la cual se vuelve compleja en la medida que se acerca al paso, y también es significada con temor por el peligro de la presencia del puma (Puma concolor) y las quilas (Chusquea quila). Además, la espesura de la selva, hace que se torne en un sitio oscuro, lo cual se entremezcla con la idea weza newen o energías desequilibrantes para los mapuches.

Este paisaje se asocia a la muerte de muchas personas (mapuches y no mapuches) que murieron en la zona durante dictadura militar intentado huir hacia Argentina. De acuerdo a los relatos, muchos de esos cuerpos desaparecieron, pero no se sabe con precisión:

La gente caía también en esas montañas y nadie sabe...yo creo que se debería hacer una investigación mucho más grande ahí antes de tratar de poner una hidroeléctrica. Tratar de recuperar cuerpos que están ahí enterrados, porque cayeron no más a esa quebrada (entrevista 2: hombre, 30 años).

Por lo tanto, el paisaje de Añihuerraqui por un lado se asocia a relatos nostálgicos y de recuperación de lugares familiares, asociados al bienestar y el descanso, y por otro lado se ha resignificado en función del miedo, la muerte provocada por la Dictadura Militar.

\section{Paisaje de Trankura}

El paisaje del Trankura emerge del tránsito hacia el interior del valle del Trankura, dentro de los límites del título de Título de Merced de Camilo Coñoequir Loftunekul, respondiendo a una movilidad más bien confinada en los márgenes de la propiedad indígena y que en el presente es dominante. Un rasgo característico de este paisaje es la topografía dominada por dos grandes cordones montañosos de origen glaciar, dispuestos en sentido N-S, en los que destacan cerros o wingkul, cada con un nombre propio en mapudungun, asignados por Lloftunekul. Los cerros del cordón este, nombrados en sentido N-S son: Kopiupulli, Pünowemañke, Peñewe, Millallifén; en el cordón W, en el sentido N-S se encuentran los cerros y volcanes: Puala, Rukañanko, Punmawe, Ketropillán (volcán Quetrupillán), Wenurangi, Awelñanko (volcán Lanín).

El cerro Pünowemañke (1.474 msm), palabra mapuche que significa "donde pisa el cóndor", es un cerro treng-treng, categoría empleada para identificar aquel wingkul que destaca por su forma y altura de las demás cumbres, que lo convierte en un hito geográfico y visual. Además, desde la cosmovisión mapuche, un treng-treng es un referente ritual dentro de la organización de los sitios ceremoniales que orientan rogativas individuales como el ngellipun, 


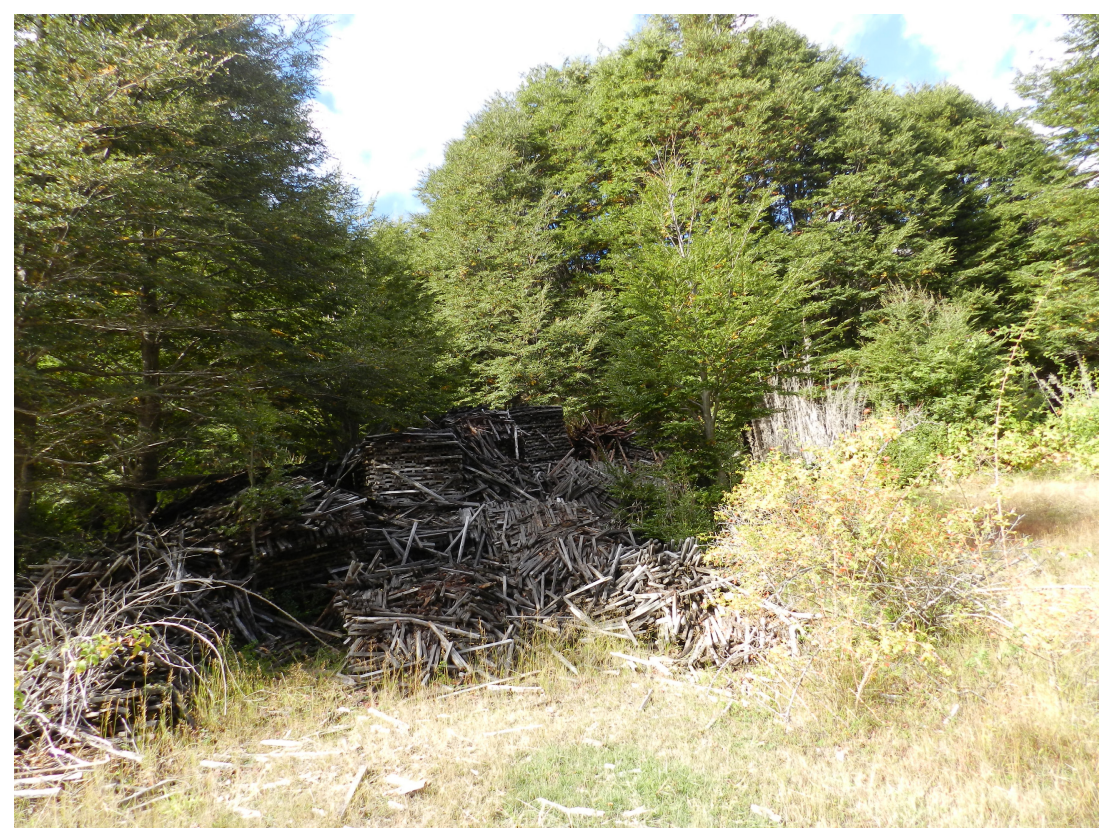

Figura 5. Parque Nacional Villarica, sector Añihuerraqui. Registro personal de la autora.

Villarrica National Park, Añihuerraqui sector. Photo credit: author.

o comunitarias como el nguillatun. Esta función explica la localización del nguillatuwe, situado a los pies del Pünowemañke, por lado occidental, el cual está en dirección hacia el treng-treng, es que el lado por donde sale el sol. Desde la memoria oral de los participantes, surgen historias que avalan el contenido religioso que tiene el cerro Pünowemañke, el cual no fue escogido al azar, si no por anuncios que recibió Lloftunekul directamente de los gnen del territorio y de sus ancestros:

Cuando a Curarrehue llega Lloftunekul, él tuvo dos nguillatuwe (...). Lo primero que hace fue a buscar un nguillatuwe porque, ¿dónde hago un nguillatun? En pewma, en rogativa, le dijeron que él ya tenía el rewe ahí. Pero era un rewe de piedra, de kura. ¿Dónde estaba este rewe? ¿Dónde estaba la fuerza de ese? Y él, en pewma -nos cuenta nuestro longko-, era el punowemañke, la roca, ese era el rewe de ellos. Ahí estaba la conexión con el wenumapu con todas las dimensiones del mundo mapuche. Y él con los nguillatufe, todo ese tiempo hicieron el nguillatun a los pies del Punowemañke, arriba del Pichitrankura y a los pies del Peñewe también. Ahí fue el nguillatun. Porque ahí está la fuerza, ahí está el newen, la conexión (entrevista 12: hombre, 22 años).

El tránsito por el Pünowemañke no es fácil. Este cerro, revestido de bosque nativo renoval de tipo caducifolio altoandino, los cuales se entremezclan con grandes rocas y afloramientos rocosos de la montaña, cuya cumbre es posible ver volar cóndores (Vultur gryphus) que anidan en este cerro. La diversidad ecológica y la presencia de agua del Pünowemañke han incentivado la habilitación de senderos gestionados por las familias mapuches del Lof Trankura. Esta iniciativa nacida de las "Rutas Ancestrales Araucaria", es una nueva forma de desplazamiento y reactivación de la movilidad en este cerro, la cual no tiene un carácter invasivo.

El cerro Peñewe (1.477 msm), llamado oficialmente por el Estado de Chile como "Cerro Lepe", cuyo nombre deriva del mapudungun y significa "mirador", también cumple una función ritual. Este cerro, emplazado al lado del Pünowemañke y separados por una quebrada por la que transita el estero Pichitrankura, también está conectado a los rituales realizados en el nguillatuwe del Lof Trankura. Esta triada de lugares significativos, configurada por el estero Pichitrankura, el cerro Pünowemañke y el cerro Peñewe, es la que ha permitido el equilibrio del territorio, siendo tres lugares relevantes del paisaje, la memoria y del bienestar del Lof Trankura, los cuales son reafirmados toda vez que realizan el nguillatun.

El cerro Millallifén, llamado comúnmente "Las Peinetas", corresponde a un cordón montañoso de $1.484 \mathrm{msm}$ aproximadamente, de origen glaciar, con grandes fisuras y cortes verticales derivados de los efectos de la erosión y meteorización (Figura 6). El nombre Millallifén es una palabra del mapudungun 
que significa "donde se va a encontrar el tesoro o riqueza", y alude a los animales vacunos salvajes o baguales que deambulaban por el lago Tromen, ubicado en las faldas orientales del Millallifén. El ganado bagual o "kullin" fue un recurso importante para los mapuches de Lof Trankura, sobre todo a principios del siglo XX. Al respecto, un entrevistado narra lo siguiente:

Milla es el tesoro que la persona se puede encontrar y llifén donde lo va a buscar. Y claro, esos son los animales salvajes que se encuentran detrás del lago Tromen. Y al encontrarse esa cantidad de animales, entonces la gente que quería armarse de animales (...) tenía que nguilleatukear no más el día antes, y en la noche soñaban: te vamos a entregar un animal en tal parte, tú vas a ir y te vamos a entregar un animal (entrevista 8: hombre, 70 años).

El volcán Quetrupillán o Ketropillán, cuyo nombre significa "volcán mocho o despuntado" es un elemento distintivo del paisaje, junto con el volcán Wenurangi, también llamado "Colmillo del diablo" o "Quinquilill", que es una prolongación del Ketropillán. Este volcán, asi como sucede con el Lanín, le es atribuido la capacidad de comunicarse con los mapuches del Lof Trankura a través de señales que ellos han aprendiendo a leer en su interacción con él. La humanización de ciertos elementos del paisaje, se entremezcla con temor y la necesidad de nguillatukear para la mantención del equilibrio de las fuerzas acumuladas en estos volcanes. Un relato grafica de mejor manera esta observación:

Sí, sí, todo lo que ocurre da un anuncio. Por ejemplo, si va haber un temblor hay muchos treiles que vuelan. Se desesperan y vuelan (...). Igual, cuando va a venir mucho nevazón, el Ketropillán hace un ruido como que van arreando animales. Entonces ahí dice el mapuche: petu ke ke cha kullin tuwi Ketropillán... "que está arreando sus animales" (entrevista 8: hombre, 70 años).

En consecuencia, la vitalidad de lo material posiciona a las fuerzas del entorno como actores sensibles con quienes se establecen relaciones de tipo social y de cuidado mutuo.

\section{Discusión}

A partir del caso del Lof Trankura, se identificaron tres niveles de análisis en relación a las narrativas de la movilidad y que gravitan en el sentido de pertenencia social del pueblo mapuche con sus

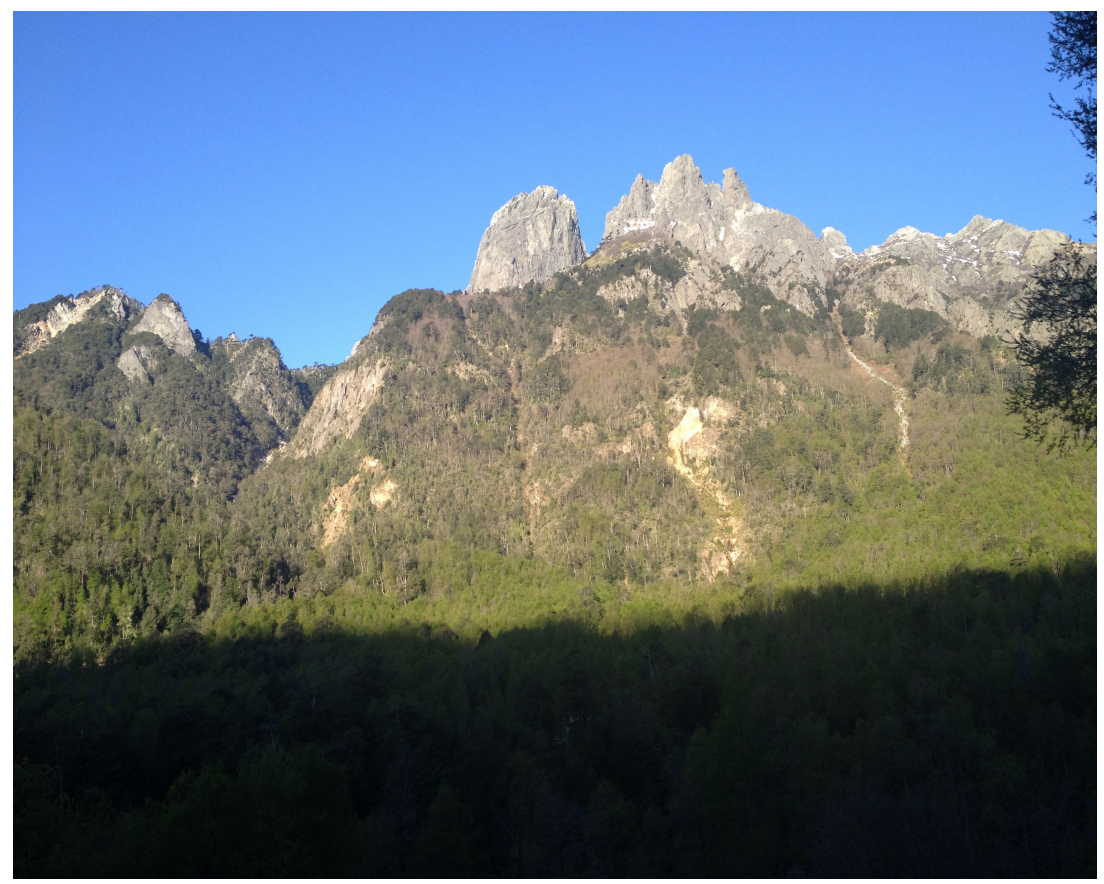

Figura 6. Cima cerro Millallifén. Registro personal de la autora.

Summit of Millallifén Hill. Photo credit: author. 
paisajes. En un primer nivel, se encuentran aquellos hitos referenciales que corresponden a sitios naturales que destacan en el paisaje, asociados a sucesos compartidos por el Lof Trankura. Pese a que muchos de estos hitos no se localicen dentro de los límites territoriales, la multisensorialidad convierte a muchos de estos elementos distantes, multiescalares e invisibles en lugares configuradores del paisaje. En un segundo nivel, están los sitios marcados e intervenidos por distintos actores y agentes: los hitos limítrofes localizados en los pasos Añihuerraqui y Paimún, el nguillatuwe del Lof Trankura, las huellas mapuches y aquellos caminos construidos por el Estado chileno. Un tercer nivel concierne a los nombres propios de cada lugar proporcionados por la toponimia mapuche o üi mapu (Melín et al. 2017), que entrega una variedad de información geográfica e interpretaciones desde el mundo mapuche.

Asumiendo que nuestras experiencias móviles en el paisaje están condicionadas por sistemas de dominación que influyen sobre dicha experiencia (Tilley 1994) es válido afirmar que las prácticas móviles del Lof Trankura se han acotado temporal y espacialmente, distorsionadas por las intervenciones estatales y las presiones económicas sobre los recursos naturales existentes en este territorio. Sin embargo, en algunos casos, son producto de los entendimientos sobre la naturaleza de los propios mapuches del Lof Trankura, la cual ha ido variando según el control y uso dado al territorio y las necesidades de las familias en determinados momentos y circunstancias. Por ejemplo, la tala de numerosas zonas boscosas realizada por los propios mapuches del Lof Trankura fue una práctica muy común durante la época de extractivismo maderero, en un contexto de pobreza y precariedad. Al contrario, hoy en día prevalecen otros valores respecto a la naturaleza, que se expresan en nuevas narrativas, prácticas y actividades económicas, dirigidas al cuidado y mantención del territorio, como el senderismo a lo largo del cerro Pünowemañke.

Los relatos y testimonios compartidos por los entrevistados están cruzadas por los afectos y emociones que influyen en cómo se vivencian, se rememoran y se narran los paisajes producidos por la movilidad. El valor emocional de la experiencia en el paisaje revela interesantes geografías del mundo vivido (Nogué 2015) que construyen y reproducen diferentes paisajes afectivos (Puente 2012) presentes en los procesos de subjetivación política que impulsan proyectos de resistencia y soberanía (GonzálezHidalgo y Zografos 2017): paisajes nostálgicos para el caso del paisaje de Mongolluko; paisajes heredados, como los antecedentes de la toponimia del valle Trancura y que refleja la propia interpretación del espacio entregada por Lloftunekul; paisajes negados y privatizados por el Estado de Chile y Argentina, quienes han limitado el uso y acceso del espacio de los valles Añihuerraqui o Mongolluko; paisajes del miedo, como la huida por la ruta del paso Añihuerraqui durante Dictadura Militar.

Las emociones también emergen desde las atribuciones humanas asignadas al paisaje, el cual se puede comunicar por diversas señales con los mapuches del Lof Trankura. Esta capacidad de comunicación con los paisajes requiere de la comprensión del mapudungun, como principal puente con el mundo invisible, sintiente y no humano, que reflejan los vínculos sociales de las personas mapuches con los paisajes (Skewes et al. 2011). También refuerza la idea de pensar los paisajes mapuches como paisajes en movimiento (Salazar et al. 2017) abandonando concepciones del paisaje que lo definen como materialidad inerte, estática, deshabitada y separada de lo humano. Por lo tanto, esta comunicación con el paisaje y sus señales demuestran la dependencia de los mapuches del Lof Trankura de sus paisajes para para poder sobrellevar peligros -por ejemplo- y asegurar la supervivencia de los mapuches en este territorio que los protege.

\section{Conclusión}

Lejos de ser simples huellas en la superficie de la tierra, sendas, senderos y caminos configuran paisajes construidos y practicados continuamente a través de la movilidad, cuyas narrativas combinan selectivamente vivencias pasadas con las percepciones presentes influidas por contextos particulares, dando forma la trama de una narración organizada en patrones espaciales que no necesariamente siguen un orden cronológico (Argounova-Low 2012; Tilley 1994). Pese a la influencia de complejos procesos modernizadores globales en territorio indígena que han constreñido las movilidades en la cordillera y sus posibilidades de recomposición, los paisajes de las movilidades mapuches involucran un continuo proceso de actualización y/o reelaboración de prácticas y narrativas de los desplazamientos que resisten y subvierten los efectos de las intervenciones de la agencia estatal en la movilidad del pueblo mapuche.

De esta manera, los paisajes de las movilidades mapuches no son paisajes neutros ni originales; más bien articulan recuerdos, emociones, corporalidad, materialidades, prácticas, conocimientos y percepciones en un continuo proceso vivo que renuevan estrategias de resistencia ante los acelerados procesos de modernización global. Esta forma de producción de subjetividades críticas realza el sentido político de los paisajes producidos por las movilidades indígenas en contextos de disputas territoriales y coloniales que 
inciden en las dimensiones discursivas del paisaje en relación a los renovados procesos de despojo que trastocan los desplazamientos cotidianos. Paisajes que articulan el pasado y el presente, reproducidos por las personas mapuches en la medida que no los olvidan, revelando la compleja geografía de un mundo vivido en la cordillera, que se reconfigura constantemente a partir de las narrativas de los desplazamientos.

Agradecimientos: Agradezco el financiamiento otorgado por la Corporación Nacional de Investigación Científica y Tecnológica (CONICYT) a través del Proyecto FONDECYT N 1130732: "Relaciones interétnicas y formación del Estado-nacional en los márgenes: sociedad, fronteras y territorio en el espacio transcordillerano Araucanía-Neuquén desde 1883", dirigido por el Dr. Álvaro Bello. Agradezco enormemente a mis lamngen del Lof Trankura, quienes me brindaron la oportunidad de conocer sus vidas en la cordillera, en especial a Simón Crisóstomo Loncopán. También agradezco al profesor Luis Curilaf por su generosa ayuda brindada en el transcurso del estudio y a los evaluadores que participaron en la revisión de este artículo por sus valiosas contribuciones. Por último, dedico este manuscrito a la memoria de Daniel Coñoequir, lawentuchefe del Lof Trankura.

\section{Referencias Citadas}

Argounova-Low, T. 2012. Narrating the road. Landscape Research 37:191-206.

Barrientos, M. 2015. Indagación sociocultural e identificación de sitios de significación cultural en la comunidad indígena Camilo Coñoequir Lloftunekul, Lof Trankura, Curarrehue. Manuscrito en posesión del autor.

Bello, A. 2000. Viaje de los mapuches de Araucanía a las pampas argentinas: una aproximación a sus significados socioculturales (siglos XIX y XX). http://www.mapuche.info/mapuint/bello0000.html

Bello, A. 2011. Nampülkafe, el Viaje de los Mapuche de La Araucanía a las Pampas Argentinas. Ediciones UCT, Temuco.

Bello, A. 2014. Cordillera, naturaleza y territorialidades simbólicas entre los mapuche del siglo XIX. Scripta Philosophiae Naturalis 6:21-33.

Benedetti, A. y E. Salizzi 2014. Fronteras en la construcción del territorio argentino. Cuadernos de Geografía: Revista Colombiana de Geografía 23:121-138.

Bengoa, J. 2000. Historia del Pueblo Mapuche: Siglo XIX y XX. Lom Ediciones, Santiago.

Capellà, H. 2009. Por los caminos de la identidad y del desarrollo regional. Atenea 500:75-90.

Careri, F. 2002. Walkscapes: El Andar como Práctica Estética. Gustavo Gili, Barcelona.

Castro, M., S. Millamán, J. Niccodemi, A. Seguel y H. Silva 2016. Los Impactos en Derechos Humanos de los Proyectos de Inversión Hidroeléctrica en el Territorio Mapuche de la Región de La Araucanía. MG Sotelo, Temuco.

Connerton, P. 1989. How Societies Remember. Cambridge University Press, Cambridge.

Cresswell, T. 2006. On the Move: Mobility in the Modern Western World. Routledge, London.

Cresswell, T. 2010. Towards a Politics of Mobility. Environment and Planning D: Society and Space 28:17-31.

Curilaf, L. 2015. Kurarrewe en el Boquete del Trankura. Cóndor Blanco Ediciones.
Di Giminiani, P. 2012. Tierras Ancestrales, Disputas Contemporáneas: Pertenencia y Demandas Territoriales en la Sociedad Mapuche Rural. Ediciones UC, Santiago.

Di Giminiani, P. y M. Fonck 2015. El paisaje como proceso de vida: experiencias de domesticación del bosque en el sur de Chile. Revista de Geografía Norte Grande 61:7-24.

Godoy, M. 2014. Las rutas del Sollipulli hacia el Puel Mapu. Revista Austral de Ciencias Sociales 27:45-69.

González-Hidalgo, M. y C. Zografos 2017. How sovereignty claims and "negative" emotions influence the process of subjectmaking: Evidence from a case of conflict over tree plantations from Southern Chile. Geoforum 78:61-73.

Grebe, E. 1993. El subsistema de los ngen en la religiosidad mapuche. Revista Chilena de Antropología 12:45-64.

Gundermann, H., H. González y L. De Ruyt 2009a. Migración y movilidad mapuche a la Patagonia Argentina. Magallania 37:21-35.

Gundermann, H., H. González y L. De Ruyt 2009b. Migración y movilidad mapuche entre Araucanía y Nor Patagonia. Avá 15:103-123.

Hernández, R., C. Fernández y P. Baptista 2006. Metodología de la Investigación. Cuarta edición, McGraw-Hill, México D.F.

Huiliñir-Curío, V. 2015. Los senderos pehuenches en Alto Biobío (Chile): articulación espacial, movilidad y territorialidad. Revista de Geografia Norte Grande 62:47-66.

Huiliñir-Curío, V. 2016. Los Paisajes de la Movilidad Mapuche en el Valle del Río Trancura, Región de La Araucanía (Chile). Tesis para optar al grado de magíster en Ciencias Sociales Aplicadas, Facultad de Educación Ciencias Sociales y Humanidades, Universidad de La Frontera, Temuco.

Huiliñir-Curío, V. y A. MacAdoo 2014. Las disputas del espacio y los senderos pehuenches en Alto Biobío. Revista Geográfica del Sur 7:95-112.

Ingold, T. 2000. The Perception of the Environment. Routledge, London.

Lindón, A. 2006. Geografías de la vida cotidiana. En Tratado de Geografía Humana, editado por D. Hiernaux y A. Lindón, pp. 477-536. Anthropos-UAM-I, Barcelona. 
Melin, M., P. Mansilla y M. Royo 2017. Mapu chillkantukun zugu: Descolonizando el mapa del Wallmapu, construyendo cartografía cultural en territorio mapuche. Manuscrito en posesión del autor.

Nogué, J. 2015. Emoción, lugar y paisaje. En Teoría y Paisaje II: Paisaje y Emoción. El Resurgir de las Geografías Emocionales, editado por L. Puigbert y G. Bretcha, pp. 137-147. Observatorio del Paisaje de Cataluña-Universidat Pompeu Fabra, Barcelona.

Núñez, A., R. Sánchez y F. Arenas 2013. Más allá de la línea: la montaña y la frontera desde su pluralidad espacio-temporal. En Fronteras en Movimiento e Imaginarios Geográficos, editado por A. Núñez, R. Sánchez y F. Arenas, pp. 25-39. Ril editores, Santiago.

Pinto, J. 1996. Integración y desintegración de un espacio fronterizo. La araucanía y las Pampas, 1550-1900. En La Araucanía y las Pampas. Un Mundo Fronterizo en América del Sur, editado por J. Pinto, pp. 11-46. Ediciones Universidad de La Frontera, Temuco.

Puente, P. 2012. El valor emocional de la experiencia paisajística. Querencias y paisajes afectivos. Cuadernos Geográficos 51:270284.

Salazar, G., M. Fonck y F. Irarrázaval 2017. Paisajes en movimiento: sentidos de lugar y prácticas interculturales en ciudades de la Región de La Araucanía, Chile. Chungara Revista de Antropología Chilena 49 (2):251-264

Skewes, J. y D. Guerra 2016. Sobre árboles, volcanes y lagos: algunos giros ontológicos para comprender la geografía mapuche cordillerana del sur de Chile. Intersecciones en Antropología 17:63-76.

Skewes, J.C., D. Guerra y C. Henríquez 2014. Patrimonio y paisaje: dos formas de ensamblar naturaleza y cultura en la cuenca del río Valdivia, sur de Chile. Chungara Revista de Antropología Chilena 46 (4):651-668.

Skewes, J.C., D. Guerra, P. Rojas y M. Mellado 2011. ¿La memoria de los paisajes o los paisajes de la memoria? Los enigmas de la sustentabilidad socioambiental en las geografías en disputa. Desenvolvimento e Meio Ambiente 23:39-57.

Tomasi, J. 2013. Espacialidades pastoriles en las tierras altoandinas. Asentamientos y movilidades en Susques, puna de Atacama (Jujuy, Argentina). Revista de Geografía Norte Grande 55:67-87.

Tilley, C. 1994. A Phenomenology of Landscape: Places, Paths, and Monuments. Berg, Oxford.

Urry, J. 2007. Mobilities. Polity Press, Cambridge.

Zavala, J. 2011. Los Mapuches del Siglo XVIII: Dinámica Interétnica y Estrategias de Resistencia. Ediciones Universidad Católica de Temuco, Temuco. 